\title{
2.2 El Currículum: una construcción social
}

Mary Cantisano Rojas ${ }^{1}$

\section{Resumen}

Una mirada histórica del concepto currículum permite ver en qué medida las circunstancias sociales, económicas, políticas e históricas de las sociedades son determinantes en la construcción social de este concepto. La historia también permite ver las implicaciones ante las diferentes teorías sobre la cuales ha podido descansar el proceso enseñanza-aprendizaje a través de los tiempos.

A mediados de los años setenta, me inscribí en la universidad. Para esa ocasión solicité al Departamento de Admisiones las informaciones correspondientes a la carrera de Trabajo Social. Me entregaron el Pensum de la carrera, documento en el cual aparecían las materias que debía cursar, el número de créditos y la duración de los estudios, entre otras informaciones.

Años después, a finales de la década de los años '80, me interesé en la docencia universitaria. Al finalizar la contratación de mis servicios como profesora de francés, la directora del departamento al que pertenecía, me precisó ciertos detalles acerca del Programa de la materia.

Más tarde, a principio de los años ‘90, en mi desempeño docente a nivel Medio, empecé a conocer la propuesta a nivel nacional de la llamada transformación curricular. Y no fue hasta el año 2002, cuando avanzaba el proyecto de mi tesis doctoral, que me encontré de frente con ese concepto fundamental del quehacer educativo que es el currículum.

Hoy día, quienes participan en diferentes encuentros educativos, hacen referencia al tema del Currículum o Plan de Estudio al poner en evidencia, de una u otra manera, la preocupación acerca del qué están haciendo las instituciones educativas con relación al perfil de los y las estudiantes y el papel que habrán de jugar y / o están jugando en la sociedad dominicana actual. En tal sentido se aprecia igualmente un interés en torno a "la calidad de la educación", "la dignificación de la educación", "retos y desafíos de la educación en el mundo de hoy", entre otros. Se escucha, "entre líneas", la preocupación de si estamos formando profesionales en las distintas ramas del saber o si estamos preparando estudiantes para ocupar un puesto de trabajo que se corresponda con demandas del mercado laboral actual.

En otras palabras, el objeto de debate es, sin llamarlo por su nombre, los Pensum, los Currículum, los Programas o los Planes de Estudios.

\section{Etimología del concepto currículum}

La historia de la problemática curricular evidencia su correspondencia con un contexto preciso en el marco de unas determinadas relaciones sociales.

La noción de currículum es muy antigua. Los trabajos de D. Hamilton y M. Gibbons (como se cita en Kemmis, 1993, p. 31) hacen referencia a los usos primitivos de este término. Etimológicamente proviene del latín y significa una pista circular de atletismo.

Para los países de habla inglesa, la noción de currículum aparece por vez primera en la universidad de Glasgow, en 1633, en relación a la organización de los cursos de estudio. Esto fue el resultado de un proceso específico de transformación de la educación en la universidad bajo la influencia de la moral calvinista.

1 Doctora en Ciencias de la Educación, en la Universidad de Paris X-Nanterre y Magister en Sociología Política, Universidad de Louvain-la-Neuve, Bélgica. Directora del Programa de Superación del Profesorado, Pontificia Universidad Católica Madre y Maestra, Recinto Santo Tomás de Aquino, Santo Domingo. 
Las universidades del medioevo seguían la estructura grecolatina y la organización de los contenidos en los conocidos Trivium (lógica, gramática y retórica) y Quadrivium (aritmética, geometría, astronomía y música). Luego, en la concepción de los Jesuitas, discipline y ratio studiorum fueron dos nociones utilizadas para designar respectivamente el orden estructural y esquemático de los estudios. Más tarde, la noción de currículum se posiciona haciendo referencia a la totalidad (el ciclo completo y a la secuencia organizada). De ahí la metáfora de progresión con una competencia de atletismo.

Ya para el siglo XIX, la problemática curricular se va alejando de lo metodológico y de las disputas religiosas y va centrando su atención en las nuevas demandas sociales y económicas del momento.
3. En el marco de la enseñanza escolástica (Santo Tomás de Aquino), el propósito era de conciliar la fe cristiana y la razón mediante el análisis de textos, la lectura y la discusión. El reto era la formación de los maestros en lo relacionado a conceptualizar todos los aspectos de la vida, lo mismo que formarlos con relación a saber hacer la síntesis de la teología cristiana, la filosofía de la antigüedad y la ciencia.

4. En la concepción de los jesuitas, lo primordial era el desarrollo de las habilidades del lenguaje y luego consagrarse al humanismo clásico: educar individuos cultivados de acuerdo a los nuevos tiempos. Los jesuitas desarrollaron la pedagogía de la rivalidad y de la competencia para motivar el aprendizaje escolar, lo mismo que estrategias para no olvidar lo aprendido.

5. El método natural (siglo XVII, Johann Amos Comenius, 1592-1670) pone espe-

"Ya para el siglo XIX, la problemática curricular se va alejando de lo metodológico y de las disputas religiosas y va centrando su atención en las nuevas demandas sociales y económicas del momento".

\section{Fundamentos del currículum: una pincelada histórica}

Broudy (citado por Kemmis, 1993, p. 34) hace un sobrevuelo histórico y presenta ocho métodos de enseñanza que ofrecen una visión de los cambios de perspectiva con relación al papel que juega la educación en la sociedad.

1. Para los sofistas el propósito de la enseñanza era enseñar a los hombres a ser elocuentes. La pedagogía tenía que ver con el desafío de entrenar a los jóvenes para que fueran exitosos en la vida política de la democracia ateniense y más tarde de la Roma republicana.

2. La dialéctica socrática buscaba no sólo el desarrollo del conocimiento, sino hacer todo lo necesario para que los alumnos se apeguen a la verdad, al bien y a la belleza como virtudes personales y sociales. cial atención al lenguaje ordinario y al conocimiento del mundo, al uso de un orden de la naturaleza que sirve como base para el aprendizaje del mundo, e insiste en la comprensión más que en la imitación. A partir de su visión perfecta de la humanidad, Comenius creía que la ilustración, mediante la educación, podía llevar a la humanidad más cerca de Dios.

6. J.H. Pestalozzi (1746-1827). Desarrolló la lección-objeto y estableció una distinción clara entre lo que es lógico y lo que es sicológico, haciendo siempre la observación de que el aprendizaje es un proceso evolutivo. Él tenía en cuenta las leyes del desarrollo del niño como base de la educación.

7. F.W.Froebel (1788-1852). Creador del kindergarten. Inspirado en la filosofía de Hegel y de la noción de unidad orgánica, según la cual, lo que sea es una totalidad 
en sí misma y una parte de la totalidad más general. Para él la educación debe ser un proceso de relaciones del saber en una unidad más amplia e incluyente.

8. La instrucción como construcción, tomado de los trabajos de J. F. Herbart (1776-1841), quien creía poder construir la experiencia sobre la base de las impresiones sensoriales y, a partir de la organización sistemática de éstas, construir los conocimientos. La teoría de la cognición estaba en la base de su perspectiva de la educación.

Es evidente que cada una de estas propuestas obedece a ideas diferentes acerca de la educación, independientemente de que en cada una se reconoce el papel que juega la educación en la sociedad. Estas diferentes perspectivas determinan lo que debe ser enseñado, aprendido, la manera de hacerlo y con quién. alternativas que podemos encontrar, cada una obedeciendo a la visión de su autor ante la situación y contexto social vividos.

En su revisión, Lundgren (1997) recoge de manera muy resumida las diversas formas en que se ha utilizado el concepto de currículum. Para unos, se refiere a un documento con un plan detallado del año escolar, es decir, como sinónimo del término programa. Para otros, es una amplia guía para los profesores o para significar todas las experiencias que un aprendiz tiene bajo la guía de la escuela.

Ahora bien, para Lundgren (1997), un currículum incluye un conjunto de principios sobre cómo deben seleccionarse, organizarse y transmitirse el conocimiento y las destrezas. Esto significa que un currículum es:

1. Una selección de contenidos y fines para la reproducción social, o sea, una

\section{"...la cuestión central del currículum es un problema de representación, es decir, que todo currículum es el producto de su época".}

Es a partir de estos aspectos, que Lundgren en su libro Teoría del currículum yescolarización (1997, p. 20), haciendo una revisión amplia del tema, considera que la cuestión central del currículum es un problema derepresentación, es decir, que todo currículum es el producto de su época. Es una construcción social en donde la actividad educativa juega un papel importante a través de sus contenidos, las ideas que se propone desarrollar, las herramientas utilizadas a favor de la reproducción y/o de la transformación de la sociedad.

\section{Definiciones de currículum}

Al parecer, toda investigación teórica pone en evidencia que no existe un punto de partida teórico-práctico estable para el estudio y la definición posterior de currículum. Esto explica la cantidad de definiciones selección de qué conocimiento y qué destrezas han de ser transmitidos por la educación.

2. Una organización del conocimiento y las destrezas.

3. Una indicación de métodos relativos.

Gimeno (1996 pp. 13-64) nos ofrece una presentación amplia en torno al currículum al exponer diferentes consideraciones de diferentes autores, como es la tesis de Rule (como se cita en Gimeno, 1996, p. 14) que reagrupa numerosas intervenciones relativas a una definición de currículum. En tal sentido, plantea que el currículum es la definición de los contenidos de la educación, de los planes o de las propuestas. Es donde se especifican los objetivos; es el reflejo de la herencia cultural; es el cambio de comportamiento, del programa de la escuela (de los contenidos y de las actividades); es el conjunto de aprendizajes o de los resultados, 
o de todas las experiencias que un niño pueda adquirir.

Forquin (como se cita en Gimeno, 1996, p, ) hace referencia a la definición ofrecida por Hirst al presentar el currículum como un programa de actividades de los docentes y del estudiantado, concebido de manera tal que estos últimos logren alcanzar en toda su dimensión ciertos fines y objetivos educativos. Otros autores lo definen de una manera más amplia, como el conjunto de los componentes de la experiencia escolar del alumno, es decir, todo lo que el estudiante aprende en la escuela y no solamente en el orden cognitivo o instrumental, sino también en el orden afectivo, social, moral y que desborda ampliamente las prescripciones oficiales y los programas explícitos.

Por último, la definición dada por D’Hainaut (1985): “un currículum es un plan de acción en el cual el desarrollo intelectual del alumno era alcanzar el equilibrio del desarrollo intelectual con la educación física (especialmente por el entrenamiento militar) y el desarrollo estético (a través de la música).

- Currículum realista. Es el resultado de la transformación del currículum clásico; los conocimientos son reforzados a través de los sentidos y lo científico.

- Currículum moral. Aparece a finales del siglo XVIII. La educación buscaba la responsabilidad de los ciudadanos con relación a los deberes del estado.

- Currículum "racional". La educación no es solamente responsable de educar a la ciudadanía, sino también de enseñar los valores liberales. Este currículum se construyó sobre la base de una filosofía pragmática, que se interesaba en el indi-

\section{"Otros autores lo definen de una manera más amplia, como... todo lo que el estudiante aprende en la escuela y no solamente en el orden cognitivo o instrumental, sino también en el orden afectivo, social, moral y que desborda ampliamente las prescripciones oficiales y los programas explícitos".}

pedagógica. Tiene que ver con las finalidades de la educación, una especificación de las actividades de enseñanza y de aprendizaje que implica el programa de contenidos y de las indicaciones precisas sobre la manera en que el profesor o el estudiante serán evaluados".

\section{Tipología de los currículum}

La tipología de los currículum obedece a los principios sobre los cuales descansa la organización y métodos seleccionados para la transmisión de los conocimientos y de las habilidades.

S. Kemmis (1993) hace la presentación siguiente:

- Currículum "clásico" de los griegos y más tarde de los romanos. Este currículo matenía un equilibrio entre la educación intelectual, física y estética viduo y en la ciencia en cuanto fundamentos de la organización racional de la sociedad.

- Currículum oculto o implícito. En éste, los aspectos de los currículum precedentes permanecen implícitos, pero los valores claves de la educación son responsabilidad de los técnicos, los especialistas del currículum; se quiere creer que se deja a los profesores y a los estudiantes un currículum neutro.

\section{Perspectivas para el análisis del concepto currículum}

Para el análisis del concepto de currículum, Gimeno (1996, pp. 36-37) propone hacerlo a partir de cinco perspectivas:

1) Desde el punto de vista de su función social, considerando su vínculo con la sociedad y la escuela; 2) En tanto proyecto

\section{Cuaderno de Pedagogía Universitaria}


o plan educativo, deseado o real, compuesto por diferentes aspectos, experiencias y contenidos; 3) Como la expresión formal y material de un proyecto, en donde se presentan los contenidos, orientaciones y secuencias; 4) En términos de un campo práctico al analizar las instrucciones y la realidad dela práctica desdela perspectiva del contenido; estudiándolo como un espacio en donde convergen diferentes prácticas que no se refieren solamente a los procedimientos de carácter pedagógico, sino a las interacciones y comunicaciones educativas; y organizando un discurso sobre la interacción entre la teoría y la práctica educativa; 5) finalmente, haciendo referencia a un determinado tipo de actividad discursiva académica y de investigación.

\section{Reflexiones finales}

En sentido general, este escenario permite de valores, supuestos y datos que se corresponden con las necesidades sociales del contexto para los cuales han sido diseñados.

- En el currículum se encuentran elementos y determinaciones muy diversas: pedagógicas, políticas, prácticas administrativas, producciones de materiales, sistemas de evaluación.

- El currículum es un instrumer\%o esencial para el mejoramiento de la calidad de la enseñanza, el cambio de condiciones de prácticas y la renovación de los establecimientos escolares.

\section{Referencias biblográficas}

D'Hainaut, L. (1985). Des fins aux objectifs. Paris: Nathan.

Gimeno, J. (1996). El currículum : una refexión

\section{"El currículum es una construcción histórica y social a través de la cual los fines de la educación se llevan a cabo, y es la base donde encontramos una teoría determinada".}

hacer las reflexiones siguientes:

- El currículum es una construcción histórica y social a través de la cual los fines de la educación se llevan a cabo, $\mathrm{y}$ es la base donde encontramos una teoría determinada.

- El currículum es la expresión de una práctica educativa, que transmite a la vez el proyecto de socialización cultural de la institución y, por tanto, de la sociedad en la cual éste se desarrolla.

- El currículum es un plan de acción. Esta acción está localizada y es elaborada a diferentes niveles.

- Los contenidos, los formatos, las actividades propuestas de los currículum corresponden a una elección históricamente determinada. Están cargados sobre la práctica, Madrid: Morata.

Kemmis, S. (1993). El currículum más allá de la teoría de la reproducción. Madrid: Morata.

Lundgren, U. (1997). Teoría del currículum y escolarización. Madrid: Morata.

Mathis, G. (1997). Professeur de français: les clés d'un savoir-faire. Paris: Nathan 